\title{
Kaon Interferometry: A Sensitive Probe of the QCD Equation of State?
}

\author{
Sven Soff ${ }^{1}$, Steffen A. Bass ${ }^{2,3}$, David H. Hardtke ${ }^{1}$, and Sergey Y. Panitkin ${ }^{4}$ \\ ${ }^{1}$ Nuclear Science Division 70-319, Lawrence Berkeley National Laboratory, 1 Cyclotron Road, Berkeley, CA94720, USA \\ ${ }^{2}$ Department of Physics, Duke University, Durham, NC27708, USA \\ ${ }^{3}$ RIKEN BNL Research Center, Brookhaven National Laboratory, Upton, NY11973, USA \\ ${ }^{4}$ Physics Department, Brookhaven National Laboratory, PO Box 5000, Upton, NY11973, USA
}

(October 24, 2018)

\begin{abstract}
We calculate the kaon interferometry radius parameters for high energy heavy ion collisions, assuming a first order phase transition from a thermalized Quark-Gluon-Plasma (QGP) to a gas of hadrons. At high transverse momenta $K_{T} \sim 1 \mathrm{GeV} / \mathrm{c}$ direct emission from the phase boundary becomes important, the emission duration signal, i.e., the $R_{\text {out }} / R_{\text {side }}$ ratio, and its sensitivity to $T_{c}$ (and thus to the latent heat) are enlarged. The QGP+hadronic rescattering transport model calculations do not yield unusually large radii $\left(R_{i} \leq 9 \mathrm{fm}\right)$. Finite momentum resolution effects have a strong impact on the extracted interferometry parameters $\left(R_{i}\right.$ and $\left.\lambda\right)$ as well as on the ratio $R_{\text {out }} / R_{\text {side }}$.
\end{abstract}

In this Letter we present predictions for the kaon interferometry measurements in $\mathrm{Au}+\mathrm{Au}$ collisions at the Relativistic Heavy Ion Collider (RHIC) (nucleon-nucleon center-of-mass energies up to $\sqrt{s}_{N N}=200 \mathrm{GeV}$ ). Correlations of identical kaon pairs as well as Bose-Einstein correlations in general represent an important tool for the understanding of the space-time dynamics in multiparticle production processes [1]. In the particular case of relativistic heavy ion collisions, one important goal is to prove the existence of a phase transition from quarkgluon matter to hadrons as predicted by QCD lattice calculations for high temperatures. Moreover, the properties of that phase transition, for example the critical temperature $T_{c}$ or its latent heat, are of great interest. For a first order phase transition, the associated large hadronization time was predicted to lead to unusually large interferometry radii [2 4 . The mixed phase of coexisting hadrons and partons prolongs the emission duration of particles from the phase boundary because of the large latent heat. This should then lead to an increase of the effective source size, in particular in the outward direction, i.e., parallel to the transverse pair velocity. This phenomenon was also expected to depend on the critical temperature $T_{c}$, the latent heat or the specific entropy of the quark-gluon phase (for recent reviews, see, e.g., [5,6]). However, as demonstrated recently [7], the subsequent hadronic rescattering phase following hadronization from a thermalized QGP does not only modify the pion interferometry radii but even dominates them. Late numerous soft collisions in the hadronic phase diminish and also alter qualitatively the particular dependencies on the QGP-properties [7].

Here, we investigate what can be learned from the interferometry analysis of kaons. The motivation to extend the interferometry analysis from pions to kaons (which are measured soon at RHIC) is provided by several as- pects. (i) Kaons are expected to be less contaminated by resonance decays compared to pions 8.97. (ii) In the case of neutral kaon correlations, two-particle Coulomb interactions do not distort the Bose-Einstein correlations (while non-negligible strong final state $\mathrm{K} \overline{\mathrm{K}}$ interactions persist due to the near threshold resonances a0(980) and f0(980) [10]). (iii) The kaon density is considerably smaller than the pion density. The pion multiplicity itself has increased by approximately $70 \%$ from SPS $\left(\sqrt{s}_{N N}=17.4 \mathrm{GeV}\right)$ to RHIC $\left(\sqrt{s}_{N N}=130 \mathrm{GeV}\right)$ 11] whereas the interferometry radii are almost the same [12,13]. Hence, higher multiparticle correlation effects that might play a role for pions, should be of minor importance for kaons. The comparison of kaon and pion correlation functions will provide a test of the presently applied two-particle correlation formalism. (iv) The strangeness distillation mechanism [14] might further increase the time delay signature $R_{\text {out }} / R_{\text {side }}$. Kaon evaporation could lead to strong temporal emission asymmetries between kaons and antikaons [15], thus probing the latent heat of the phase transition.

We will show in the following that for kaons, as for the pions, the bulk properties of the two-particle correlation functions are dominated by a long-lived hadronic rescattering phase. Thus, the interferometry radii appear to depend only weakly on the precise properties of the QGP, such as the thermalization time $\tau_{i}, T_{c}$, the latent heat or the specific entropy of the QGP. However, we will demonstrate that this sensitivity is considerably enlarged at high transverse momenta $K_{T} \sim 1 \mathrm{GeV} / \mathrm{c}$. In this kinematic regime, direct emission from the phase boundary becomes important $(\sim 30 \%)$ allowing us to inspect the prehadronic phase with less distortions. Finally, we will calculate explicitly the correlation functions and extract the corresponding parameters with and without taking finite momentum resolution effects into account. A strong impact on the radii, the $\lambda$ intercept parameter and the $R_{\text {out }} / R_{\text {side }}$ ratio are observed; they all decrease, depending on $K_{T}$, if a finite momentum resolution is taken into account.

The calculations are performed within the framework of a relativistic transport model that describes the initial dense phase of a QGP by means of ideal hydrodynamics employing a bag model equation of state that exhibits a first order phase transition [4, 16]. Hence, we focus on a phase transition in local equilibrium, proceeding through the formation of a mixed phase. Smaller radii and emis- 
sion times may result for a crossover [4,17 or for a rapid out-of-equilibrium phase transition similar to spinodal decomposition [18]. Cylindrically symmetric transverse expansion and longitudinally boost-invariant scaling flow are assumed [4,16,19]. This approximation should be reasonable for central collisions at high energy, and around midrapidity. The model reproduces the measured $p_{T^{-}}$ spectra and rapidity densities of a variety of hadrons at $\sqrt{s}_{N N}=17.4 \mathrm{GeV}$ (CERN-SPS energy), when assuming the standard thermalization (proper) time $\tau_{i}=1 \mathrm{fm} / \mathrm{c}$, and an entropy per net baryon ratio of $s / \rho_{B}=45$ [19,20]. Due to the higher density at midrapidity, thermalization may be faster at BNL-RHIC energies - here we assume $\tau_{i}=0.6 \mathrm{fm} / \mathrm{c}$ and $s / \rho_{B}=200$. (With these initial conditions results on the multiplicity, the transverse energy, the $p_{T}$-distribution of charged hadrons, and the $\bar{p} / p$ ratio are described quite well [19]20].) The later hadronic phase is modeled via microscopic transport that allows us to calculate the so-called freeze-out, i.e., the time and coordinate space points of the last strong interactions of an individual particle species, rather than applying a freeze-out prescription as necessary in the pure hydrodynamic approach. Here, we employ a semi-classical transport model that treats each particular hadronic reaction channel (formation and decay of hadronic resonance states and $2 \rightarrow n$ scattering) explicitly 21]. The transition at hadronization is performed by matching the energy-momentum tensors and conserved currents of the hydrodynamic solution and of the microscopic transport model, respectively (for details, see 20]). The microscopic model propagates each individual hadron along a classical trajectory, and performs $2 \rightarrow n$ and $1 \rightarrow m$ processes stochastically. Meson-meson and meson-baryon cross sections are modeled via resonance excitation and also contain an elastic contribution. All resonance properties are taken from [22]. The $\pi K$ cross section for example is either elastic or is dominated by the $K^{*}(892)$, with additional contributions from higher energy states. In this way, a good description of elastic and total kaon cross sections in vacuum is obtained [21]. Medium effects on the hadron properties, as for example recently studied by hydrodynamical calculations employing a chiral equation of state [17], are presently neglected. For further details of this dynamical two-phase transport model, we refer to refs. 19,20.

For the correlation analysis, a coordinate system is used in which the long axis $(z)$ is parallel to the beam axis, where the out direction is parallel to the transverse momentum vector $\mathbf{K}_{\mathbf{T}}=\left(\mathbf{p}_{\mathbf{1} \mathbf{T}}+\mathbf{p}_{\mathbf{2} \mathbf{T}}\right) / 2$ of the pair, and the side direction is perpendicular to both. Thus, $R_{\text {out }}$ probes the spatial and temporal extension of the source while $R_{\text {side }}$ only probes the spatial extension. Thus the ratio $R_{\text {out }} / R_{\text {side }}$ gives a measure of the emission duration (see also eqs.(1)-(3) and discussion below). It has been suggested that the ratio $R_{\text {out }} / R_{\text {side }}$ should increase strongly once the initial entropy density $s_{i}$ becomes substantially larger than that of the hadronic gas at $T_{c}$ 顿. The Gaussian radius parameters are obtained from a saddle-point integration over the classical phase space distribution of the hadrons at freeze-out that is identified with the Wigner density of the source, $S(x, K)$ [24,66, 23].

$$
\begin{aligned}
R_{\text {side }}^{2}\left(\mathbf{K}_{\mathbf{T}}\right) & =\left\langle\tilde{y}^{2}\right\rangle\left(\mathbf{K}_{\mathbf{T}}\right), \\
R_{\text {out }}^{2}\left(\mathbf{K}_{\mathbf{T}}\right) & =\left\langle\left(\tilde{x}-\beta_{t} \tilde{t}\right)^{2}\right\rangle\left(\mathbf{K}_{\mathbf{T}}\right)=\left\langle\tilde{x}^{2}+\beta_{t}^{2} \tilde{t}^{2}-2 \beta_{t} \tilde{x} \tilde{t}\right\rangle, \\
R_{\text {long }}^{2}\left(\mathbf{K}_{\mathbf{T}}\right) & =\left\langle\left(\tilde{z}-\beta_{l} \tilde{t}\right)^{2}\right\rangle\left(\mathbf{K}_{\mathbf{T}}\right),
\end{aligned}
$$

with $\tilde{x}^{\mu}\left(\mathbf{K}_{\mathbf{T}}\right)=x^{\mu}-\left\langle x^{\mu}\right\rangle\left(\mathbf{K}_{\mathbf{T}}\right)$ being the spacetime coordinates relative to the momentum dependent effective source centers. The average in (11)-(3) is taken over the emission function, i.e. $\langle f\rangle(K)=$ $\int d^{4} x f(x) S(x, K) / \int d^{4} x S(x, K)$ with $K=\left(E_{K}, \mathbf{K}\right)$. In the osl system $\beta=\left(\beta_{t}, 0, \beta_{l}\right)$, where $\beta=\mathbf{K} / E_{K}$ and $E_{K}=\sqrt{m^{2}+\mathbf{K}^{2}}$. Below, we cut on midrapidity kaons $(|y|<0.5)$. In the absence of $\tilde{x}-\tilde{t}$ correlations, i.e. in particular at small $K_{T}$, a large duration of emission $\Delta \tau=$ $\sqrt{ }\left\langle\tilde{t}^{2}\right\rangle$ increases $R_{\text {out }}$ relative to $R_{\text {side }}$ [2]. For strong (positive) $\tilde{x}-\tilde{t}$-correlations or large spatial anisotropies in out- and side-direction $\left.\left(\left\langle\tilde{y}^{2}\right\rangle\right\rangle\left\langle\tilde{x}^{2}\right\rangle\right)$, in principle $R_{\text {out }} \leq R_{\text {side }}$ could follow.

The absolute values of the kaon radii determined by the above expressions (11)-(3) are considerably smaller than the pion radii, especially at low $K_{T}$. These pion radii are larger by a factor of two at low $K_{T}(\leq 400 \mathrm{MeV})$ while at higher $K_{T}$ the values become similar. This is due to the resonance source character of pions. Microscopic transport calculations show that at SPS energies $\left(\sqrt{s}_{N N}=17.4 \mathrm{GeV}\right)$ about $80 \%$ of the pions are emitted from various resonances [25]. This leads to a strong substructure of the freeze-out distributions [25], e.g. strongly non-Gaussian tails. The ratio $R_{\text {out }} / R_{\text {side }}$ for kaons is shown in Fig. 1. Due to identical freeze-out distributions the correlation parameters are the same for kaons and antikaons. The bag parameter $B$ is varied from $380 \mathrm{MeV} / \mathrm{fm}^{3}$ to $720 \mathrm{MeV} / \mathrm{fm}^{3}$, (i.e., the latent heat changes by $\sim 4 B$ ), corresponding to critical temperatures of $T_{c} \simeq 160 \mathrm{MeV}$ and $T_{c} \simeq 200 \mathrm{MeV}$, respectively. A change of $T_{c}$ implies a variation of the longitudinal and transverse flow profiles on the hadronization hypersurface (which is the initial condition for the subsequent hadronic rescattering stage). $R_{\text {out }} / R_{\text {side }}$ is smaller for kaons than for pions at the same small $K_{T}$ because of their larger mass (leading to smaller velocities which reduce the temporal contribution to $R_{\text {out }}$ in eq. (2) $\left(\beta_{t}^{2}\left\langle\tilde{t}^{2}\right\rangle\right)$ [26]). The ratios reach a value of 1.5 at $K_{T} \approx m_{\pi}$ and $K_{T} \approx m_{\mathrm{K}}$ for the rather rapidly rising pions [7] and the gradually growing kaons, respectively. Model calculations solely based on hadronic degrees of freedom generally predict smaller ratios [27,9, 28]. The sensitivity of the value of $R_{\text {out }} / R_{\text {side }}$ to the critical temperature $T_{c}$ increases strongly with $K_{T}$. Higher $T_{c}$ speeds up hadronization but on the other hand prolongs the dissipative hadronic phase that dominates the radii. Moreover, in the lower $T_{c}$ case, direct emission and immediate 
freeze-out from the phase boundary becomes important at large $K_{T}(\sim 1 \mathrm{GeV} / \mathrm{c})$. High- $K_{T}$ kaons are strongly correlated with early mean emission times. The resonance contribution for the kaons is still quite large, decreasing with $K_{T}$ from 70 to $50 \%$ for $T_{c} \simeq 160 \mathrm{MeV}$. However, most of these kaons are from $K^{*}(892)$ decays with the $K^{*}$ having a moderate lifetime of $\tau \approx 4 \mathrm{fm} / \mathrm{c}$. Elastic scatterings prior to freeze-out contribute on the order of $20 \%$. The direct emission from the phase boundary, i.e., the kaon did not suffer further collisions in the hadron gas after the particle had hadronized, increases strongly (approximately linearly with $K_{T}$ ) for $T_{c} \simeq 160 \mathrm{MeV}$ up to $30 \%$ at $K_{T}=1 \mathrm{GeV} / \mathrm{c}$. For the higher $T_{c}(\simeq 200 \mathrm{MeV})$ hadronization is earlier. The hadronic phase lasts longer and the system is rather opaque for direct emission. This direct emission component is not present in pure ideal hydrodynamical calculations (e.g. 26]) for which all particles, also at high $K_{T}$, are in (local) thermodynamical equilibrium. Thus, there is no possibility for direct emission from the phase boundary and escaping the hadronic phase unperturbed.

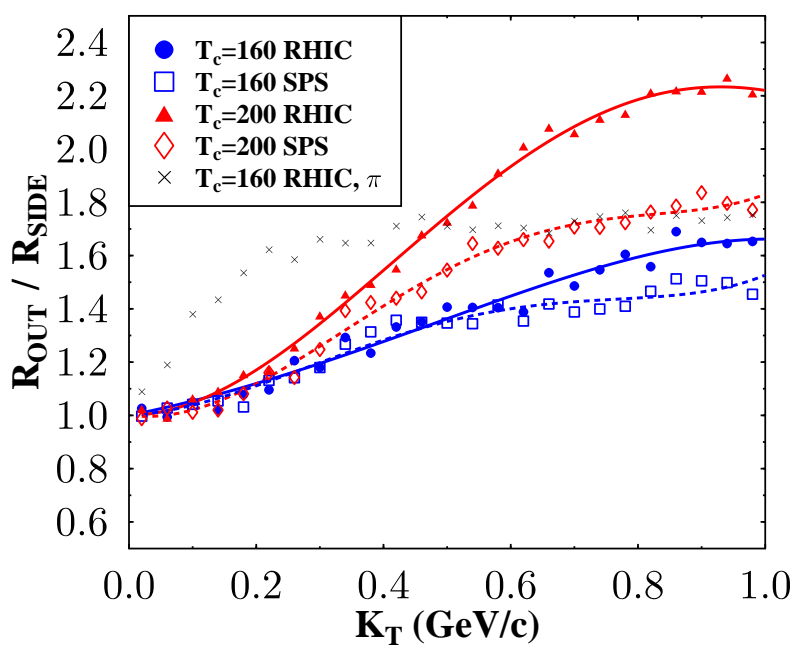

FIG. 1. $R_{\text {out }} / R_{\text {side }}$ as obtained from eqs. (1) and (2) for kaons at RHIC $\left(\sqrt{s}_{N N}=200 \mathrm{GeV}\right)$ (full symbols) and at SPS $\left(\sqrt{s}_{N N}=17.4 \mathrm{GeV}\right)$ (open symbols), as a function of $K_{T}$ for critical temperatures $T_{c} \simeq 160 \mathrm{MeV}$ and $T_{c} \simeq 200 \mathrm{MeV}$, respectively. The crosses show pions for comparison. The lines are to guide the eye.

Finally, we calculate the correlation parameters (shown in Fig. 2) by performing a $\chi^{2}$ fit of the three-dimensional correlation function $C_{2}\left(q_{\text {out }}, q_{\text {side }}, q_{\text {long }}\right)$ to a Gaussian as

$$
C_{2}\left(q_{\mathrm{o}}, q_{\mathrm{s}}, q_{\mathrm{l}}\right)=1+\lambda \exp \left(-q_{\mathrm{o}}^{2} R_{\mathrm{o}}^{2}-q_{\mathrm{s}}^{2} R_{\mathrm{s}}^{2}-q_{\mathrm{l}}^{2} R_{\mathrm{l}}^{2}\right) .
$$

The correlation functions are calculated from the phase space distributions of kaons at freeze-out using the correlation after burner by Pratt [2,23]. It is assumed that the particles are emitted from the large system independently, which allows to factorize the $N$-boson production amplitude into $N$ one-boson amplitudes $\mathcal{A}(x)$. Then, the emission function is computed as the Wigner transform $S(x, K)=\int \mathrm{d}^{4} y e^{i y \cdot K} \mathcal{A}^{*}(x+y / 2) \mathcal{A}(x-y / 2)$. The twoboson correlation function is given by

$$
\begin{aligned}
& C_{2}(\mathbf{p}, \mathbf{q})-1= \\
& \frac{\int \mathrm{d}^{4} x S(x, \mathbf{K}) \int \mathrm{d}^{4} y S(y, \mathbf{K}) \exp (2 i k \cdot(x-y))}{\int \mathrm{d}^{4} x S(x, \mathbf{p}) \int \mathrm{d}^{4} x S(y, \mathbf{q})} \\
\simeq & \frac{\int \mathrm{d}^{4} x S(x, \mathbf{K}) \int \mathrm{d}^{4} y S(y, \mathbf{K}) \exp (2 i k \cdot(x-y))}{\left|\int \mathrm{d}^{4} x S(x, \mathbf{K})\right|^{2}}
\end{aligned}
$$

where $2 \mathbf{K}=\mathbf{p}+\mathbf{q}, 2 \mathbf{k}=\mathbf{p}-\mathbf{q}$, and $2 k^{0}=E_{p}-E_{q}$. The second line in (5) holds in the limit where the width of the correlation function is small such that $\mathbf{p} \sim \mathbf{q} \sim \mathbf{K}$.

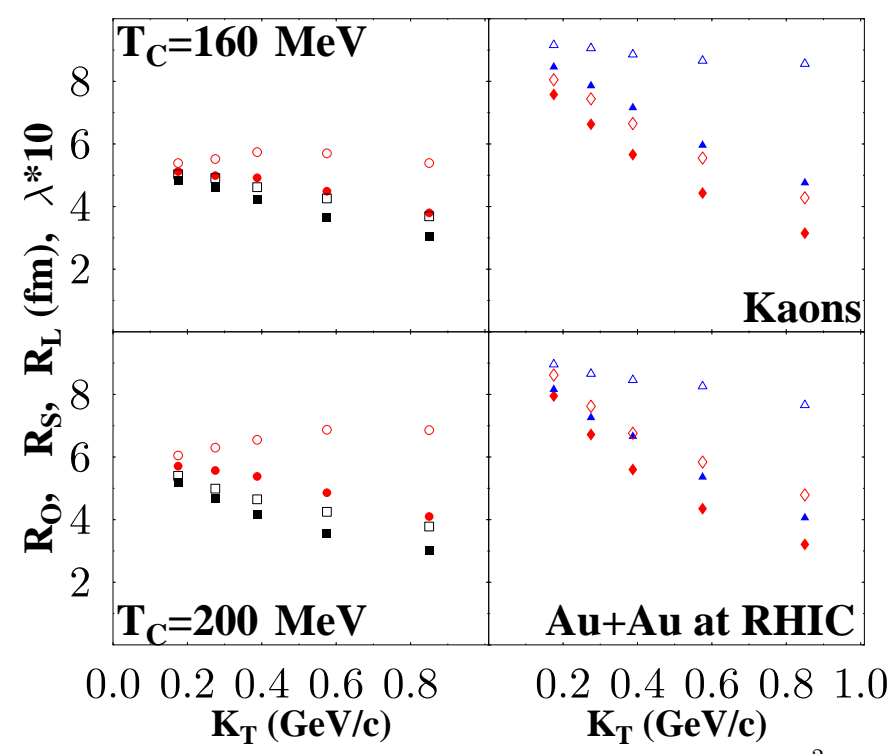

FIG. 2. Kaon correlation parameters as obtained from a $\chi^{2}$ fit of $C_{2}$ (eq. (5)) to the Gaussian ansatz (eq. (4)) for $\mathrm{Au}+\mathrm{Au}$ collisions at RHIC $\left(\sqrt{s}_{N N}=200 \mathrm{GeV}\right)$. The top and bottom panels show the results for $T_{c} \simeq 160 \mathrm{MeV}$ and $T_{c} \simeq 200 \mathrm{MeV}$, respectively. $R_{\text {out }}$ (circles) and $R_{\text {side }}$ (squares) are shown on the left while $R_{\text {long }}$ (diamonds) and $\lambda \cdot 10$ (triangles) are shown on the right. Full and open symbols correspond to calculations with and without taking momentum resolution effects into account, respectively.

Given a model for a chaotic source described by $S(x, \mathbf{K})$, such as the transport model described above, eq. (5) can be employed to compute the correlation function. The expressions (11)-(3), based on an Gaussian ansatz, yield larger values for the pion radii than performing a fit to the correlation functions. For the kaon transverse radii, similar results are obtained with both methods. Only $R_{\text {long }}$ is larger if determined by (3) as compared to the fitting result. This is due to the nonGaussian contribution arising from the longitudinal expansion dynamics that is similar for pions and kaons [28]. Kaons are better candidates for the Gaussian expressions. There are fewer resonance decays into kaons compared to pions, and long-lived resonances do not play as important 
a role.

For $T_{c} \simeq 200 \mathrm{MeV}, R_{\text {out }}$ is only approximately $1 \mathrm{fm}$ larger than in the $T_{c} \simeq 160 \mathrm{MeV}$ case. This reflects a fact already known from the pions. Higher $T_{c}$ leads to an earlier hadronization, thus causing a prolonged hadronic phase. When taking finite momentum resolution (f.m.r.) into account, the true particle momentum $p$ obtains an additional random component. This random component is assumed to be Gaussian with a width $\delta p$. The relative momenta of pairs are then calculated from these modified momenta. However, the correlator is calculated with the true relative momentum. While $R_{\text {out }}$ remains constant or even slightly increases with $K_{T}$ when calculated without f.m.r., it drops if a f.m.r. of $\approx 2 \%$ of the center of each $K_{T}$ bin is considered, a value assumed for the STAR detector 12]. Accordingly, the differences with and without f.m.r. increase with $K_{T}$. The f.m.r. leads to smaller radii. $R_{\text {out }}$ is strongly reduced while $R_{\text {side }}$ shows a moderate reduction. Thus, the $R_{\text {out }} / R_{\text {side }}$ ratio is considerably reduced through the f.m.r. For example, in the $T_{c} \simeq 200 \mathrm{MeV}$ case, it is reduced from 1.8 to 1.35. However, it is always larger than one. A proper correction for f.m.r. (STAR and other experiments 29] do correct) is needed to maintain the sensitivity to the QGP-properties. The $\lambda$ parameter is roughly constant as function of $K_{T}$ for $\delta p / p=0$ but it decreases rapidly with a f.m.r.. The correlation strength is transported to larger $q$ values by the f.m.r. effects.

We have calculated kaon correlation parameters for $\mathrm{Au}+\mathrm{Au}$ collisions at RHIC energies, assuming a firstorder phase transition from a thermalized QGP to a gas of hadrons. No unusually large radii are seen $\left(R_{i} \leq\right.$ $9 \mathrm{fm})$. A strong direct emission component from the phase boundary is found at high transverse momenta $\left(K_{T} \sim 1 \mathrm{GeV} / \mathrm{c}\right)$ where also the sensitivity to the critical temperature, the latent heat and specific entropy of the QGP is enlarged. Finite momentum resolution effects reduce the true parameters $\left(R_{i}, \lambda\right)$ and the ratio $R_{\text {out }} / R_{\text {side }}$ substantially. Kaon results from RHIC at high $K_{T}$ will provide an excellent probe of the space-time dynamics close to the phase-boundary and to the properties of this prehadronic state, possibly an equilibrated Quark-Gluon-Plasma.

\section{ACKNOWLEDGMENTS}

We are grateful to A. Dumitru, M. Gyulassy, M. Lisa, L. McLerran, S. Pratt, D.H. Rischke, R. Snellings, H. Stöcker, X.N. Wang, and N. Xu for many valuable comments. We thank the UrQMD collaboration for permission to use the UrQMD transport model and S. Pratt for providing the correlation program CRAB. S.S. has been supported by the Alexander von Humboldt Foundation and DOE Grant No. DE-AC03-76SF00098. S.A.B. acknowledges support from DOE Grant No. DE-FG0296ER40945 and DE-AC02-98CH10886.
[1] G. Goldhaber et al., Phys. Rev. 120, 300 (1960); G. I. Kopylov, M. I. Podgoretsky, Sov. J. Nucl. Phys. 15, 219 (1972); E. Shuryak, Phys. Lett. B 44, 387 (1973); G. I. Kopylov, Phys. Lett. B 50, 472 (1974); M. Gyulassy et al., Phys. Rev. C 20, 2267 (1979); A. Makhlin, Y. Sinyukov, Z. Phys. C 39, 69 (1988); Y. Hama, S. Padula, Phys. Rev. D 37, 3237 (1988); M. I. Podgoretsky, Sov. J. Part. Nucl. 20, 266 (1989).

[2] S. Pratt, Phys. Rev. D 33, 1314 (1986); G. Bertsch, M. Gong, M. Tohyama, Phys. Rev. C 37, 1896 (1988).

[3] B. R. Schlei et al., Phys. Lett. B 293, 275 (1992); J. Bolz et al., Phys. Rev. D 47, 3860 (1993).

[4] D. Rischke, M. Gyulassy, Nucl. Phys. A608, 479 (1996).

[5] R. M. Weiner, Phys. Rept. 327, 249 (2000); T. Csörgö, hep-ph/0001233, G. Baym, Acta Phys. Polon. B 29, 1839 (1998).

[6] U. Wiedemann, U. Heinz, Phys. Rept. 319, 145 (1999).

[7] S. Soff et al., Phys. Rev. Lett. 86, 3981 (2001).

[8] M. Gyulassy, S. Padula, Phys. Rev. C 41, 21 (1990); Phys. Lett. B 217, 181 (1989).

[9] J. P. Sullivan et al., Phys. Rev. Lett. 70, 3000 (1993).

[10] R. Lednicky, V. L. Lyuboshits, Sov. J. Nucl. Phys. 35, 770 (1982); Y. Sinyukov et al., Phys. Lett. B 432, 248 (1998).

[11] PHOBOS Collaboration, B. B. Back et al., Phys. Rev. Lett. 85, 3100 (2000); nucl-ex/0108009

[12] STAR Collaboration, C. Adler et al., Phys. Rev. Lett. 87, 082301 (2001); S. Panitkin, nucl-ex/0106018.

[13] PHENIX Collaboration, S. C. Johnson et al., Nucl. Phys. A698, 603 (2002); W. A. Zajc et al., nucl-ex/0106001.

[14] C. Greiner et al., Phys. Rev. Lett. 58, 1825 (1987); C. Spieles et al., Phys. Rev. Lett. 76, 1776 (1996).

[15] S. Soff et al., J. Phys. G 23, 2095 (1997); D. Ardouin et al., Phys. Lett. B 446, 191 (1999).

[16] H. Von Gersdorff et al., Phys. Rev. D 34, 2755 (1986).

[17] D. Zschiesche et al., nucl-th/0107037.

[18] T. Csörgő, L.P. Csernai, Phys. Lett. B 333, 494 (1994); H. Heiselberg, A. D. Jackson, nucl-th/9809013; J. Rafelski, J. Letessier, Phys. Rev. Lett. 85, 4695 (2000); A. Dumitru, R. Pisarski, Phys. Lett. B 504, 282 (2001); O. Scavenius et al., Phys. Rev. Lett. 87, 182302 (2001).

[19] A. Dumitru and D. Rischke, Phys. Rev. C 59, 354 (1999).

[20] S. A. Bass, A. Dumitru, Phys. Rev. C 61, 064909 (2000).

[21] S. A. Bass et al., Prog. Part. Nucl. Phys. 41, 255 (1998); M. Bleicher et al., J. Phys. G 25, 1859 (1999).

[22] Review of Particle Physics, Eur. Phys. J. C 3, 1 (1998).

[23] S. Pratt et al., Phys. Rev. C 42, 2646 (1990); S. Pratt, Phys. Rev. Lett. 53, 1219 (1984); Phys. Rev. C 49, 2722 (1994); W. A. Zajc, Phys. Rev. D 35, 3396 (1987); S. Pratt et al., Nucl. Phys. A566, 103c (1994).

[24] M. I. Podgoretsky, Sov. J. Nucl. Phys. 37, 272 (1983).

[25] S. Soff et al., CRIS'98, Acicastello, Italy, 1998; proceedings Measuring the size of things in the universe: HBT interferometry and heavy ion physics; edited by S. Costa et al., World Scientific, (1999), 221-233.

[26] S. Bernard et al., Nucl. Phys. A 625, 473 (1997).

[27] D. E. Fields et al., Phys. Rev. C 52, 986 (1995).

[28] D. Hardtke, S. Voloshin, Phys. Rev. C 61, 024905 (2000).

[29] NA44 Collaboration, I. G. Bearden et al., Phys. Rev. 
Lett. 87, 112301 (2001). 